\title{
Varying Phases of Alumina Nanowires Templated by Vertically Aligned Carbon Nanotubes Grown via Atomic Layer Deposition
}

\author{
Lester Lampert ${ }^{1}$, Sean Smith ${ }^{2}$, Brittany Timonen ${ }^{1}$, John F. Conley, Jr. ${ }^{2}$, and Jun Jiao ${ }^{1}$ \\ ${ }^{1 .}$ Department of Physics and Department of Mechanical \& Materials Engineering, Portland State \\ University, Portland, OR, USA. \\ 2. School of Electrical Engineering \& Computer Science, Oregon State University, Corvallis, OR, USA.
}

Typically, alumina nanowires (NWs) are synthesized via physical evaporation, templated anodized aluminum oxide (AAO), or the vapor-liquid-solid (VLS) process [1,2,3]. All of these techniques have been shown to demonstrate successful growth of alumina NWs; however, in the cases where a facile method resulting in vertically aligned NWs is desired, many of these methods are difficult to integrate into a fabrication process. Physical evaporation results in randomly aligned NWs and requires high temperatures therefore mitigating the possibility to use amorphous alumina and $\gamma$-alumina, among other low-temperature crystalline phase transitions [1]. And with the VLS process, expensive catalysts such as gold and germanium are used and the resulting alumina NWs exhibit a hierarchical organization with groups of many NWs growing from a single catalyst particle [2]. Lastly, templated AAO is a method that can provide vertically aligned NWs with high yield, however, in practice etching methods for creating structures with a high degree of physical dimension control is difficult to optimize [3]. Here, we present a method for creation of vertically aligned alumina NWs using vertically aligned carbon nanotubes (CNTs) as a template during atomic layer deposition (ALD). Post deposition, the deposited alumina can undergo crystalline phase changes via thermal processing within an oxygen-rich environment.

CNTs were grown by first depositing $3.5 \mathrm{~nm}$ of nickel (Ni) catalyst via magnetron sputtering onto a silicon wafer with $100 \mathrm{~nm}$ of $\mathrm{SiO}_{2}$ thermal oxide. Next, the wafers with the thin film of Ni were placed into a plasma enhanced chemical vapor deposition (PECVD) chamber and rapidly heated to $800{ }^{\circ} \mathrm{C}$ within a reducing environment provided by a $200 \mathrm{sccm}$ flow of $\mathrm{NH}_{3}$ Once at the desired growth temperature, $50 \mathrm{sccm}$ of $\mathrm{C}_{2} \mathrm{H}_{2}$ was delivered into the chamber until the growth process was terminated. For ALD deposition of amorphous alumina, the as-grown CNTs (Figure 1a) were placed into a flowthrough hot-wall ALD reactor (Arradiance Gemstar) and maintained at $175^{\circ} \mathrm{C}$ during 191 cycles of 500 ms pulses of trimethylaluminum (TMA) and $\mathrm{H}_{2} \mathrm{O}$ separated by $40 \mathrm{~s}$ nitrogen purges for an ultimate thickness of $25 \mathrm{~nm}$. Both phases, $\gamma$ and $\alpha$, of alumina were achieved by thermal annealing in a tube furnace at 10 mTorr with $20 \mathrm{sccm}$ of $\mathrm{O}_{2}$ at $950{ }^{\circ} \mathrm{C}$ and $1200{ }^{\circ} \mathrm{C}$, respectively. Scanning electron microscopy (SEM) and transmission electron microscopy (TEM) images were acquired with an FEI Sirion FESEM and an FEI Tecnai F20 TEM, respectively. Electron diffraction images were attained with the FEI Tecnai F20 TEM and selected area aperture (SAE).

Our results demonstrate the growth of vertically aligned amorphous, $\gamma$-phase, and $\alpha$-phase alumina NWs (Fig. 2a, 2b, and 2c). Amorphous alumina NWs exhibit a rough surface and sharp tips (Figure 2d) while the first thermal annealing process caused the surfaces to become smoother and transition to $\gamma$-phase alumina as revealed through electron diffraction (Figure 2e). Upon the final annealing at $1200{ }^{\circ} \mathrm{C}$, the $\gamma$ phase alumina NWs transitioned to $\alpha$-phase as indicated by electron diffraction (Figure $2 \mathrm{f}$ ). After the initial annealing of the amorphous alumina NWs within an $\mathrm{O}_{2}$ environment at $950{ }^{\circ} \mathrm{C}$, the $\mathrm{CNTs}$ reacted 
with the $\mathrm{O}_{2}$ and degrade their covalent bonds as the temperature had exceeded $400{ }^{\circ} \mathrm{C}$ leaving hollow alumina NWs with a maintained vertical alignment.

\section{References:}

[1] X. S. Fang et al, Journal of Physics: Cond. Matter 16 (2004), p. 4157.

[2] Z. Gu et al, Nanoscale 1 (2009), p. 347.

[3] Y. T. Pang et al, J Solid State Electrochem 7 (2003), p. 344

[4] The authors acknowledge support from the Maseeh College of Engineering at Portland State University.
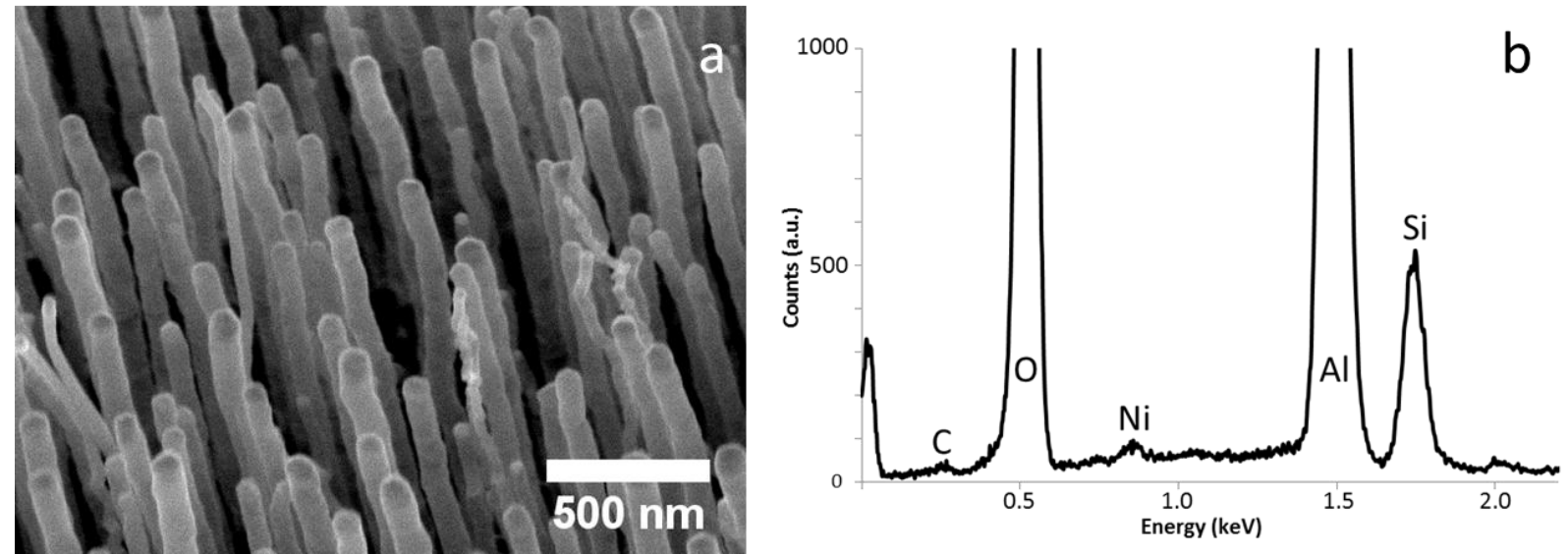

Figure 1. (a) SEM image of as-grown vertically aligned carbon nanotubes synthesized via plasma enhanced chemical vapor deposition, (b) EDS spectrum from initial coating of amorphous alumina by atomic layer deposition.
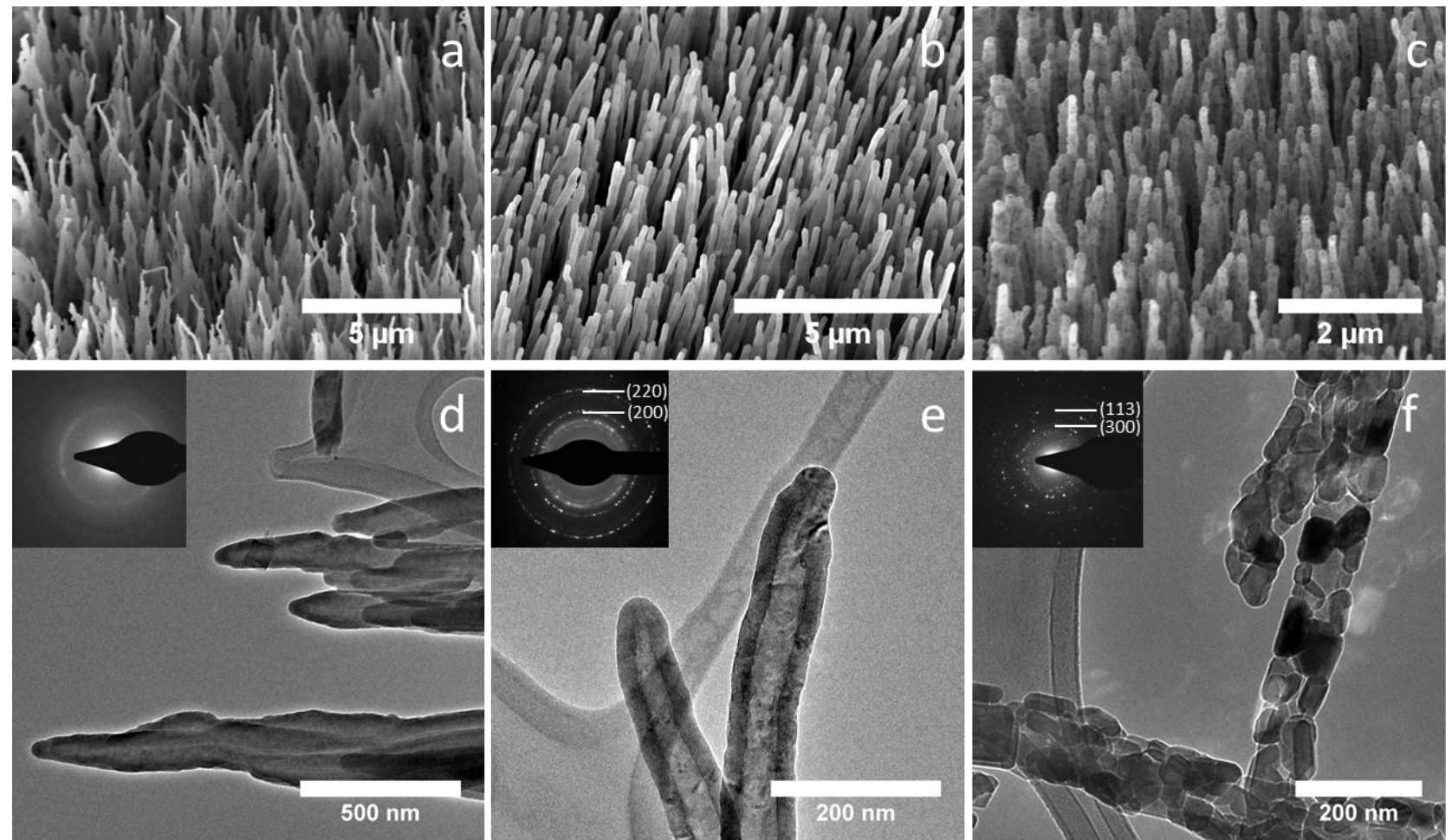

Figure 2. (a,b,c) SEM images of vertically aligned alumina nanowire arrays with amorphous, $\gamma$, and $\alpha$ crystalline phases, respectively, $(\mathrm{d}, \mathrm{e}, \mathrm{f}) \mathrm{TEM}$ images with inserted electron diffraction images of amorphous, $\gamma$, and $\alpha$ crystalline phase alumina nanowires, respectively. 